\title{
LncRNA Gm12664-001 ameliorates nonalcoholic fatty liver through modulating miR-295-5p and CAV1 expression
}

\author{
Qiao Zhang ${ }^{1,2}$, Jiemei Wang ${ }^{1}$, Hongyin $\mathrm{Li}^{1}$, Yuan Zhang ${ }^{1}$, Xia Chu' ${ }^{1}$ Jianjun Yang ${ }^{3}$ and Ying $\mathrm{Li}^{1^{*}}$ (D)
}

\begin{abstract}
Background: Our study aims to investigate the mechanisms of IncRNA Gm12664-001 improved hepatic lipid accumulation-initiated NAFLD via regulating miR-295-5p and CAV1 in AML12 cells.

Methods: The animals were divided into normal control (NC) group and high fat diet (HFD) group (20 mice per group) for $8 \mathrm{w}$. The steatotic liver was measured by hematoxylin eosin (HE) staining and kits. We performed systematical analyses on hepatic expression profiles of long noncoding RNAs (IncRNAs) and microRNAs in a high-fat diet (HFD)-induced steatotic animal model. The expression profile of targets was confirmed by bioinformatics analysis, luciferase assay, RT-PCR and western blot in AML12 cells.

Results: HFD treatment markedly observed hepatic fatty degeneration with primarily fat vacuoles, and increased TG level compared with control. According to microarray data, we found that transfection of Gm12664-001 siRNA (siRNA-118,306) obviously enhanced TG accumulation and repressed CAV1 in AML12 cells. Furthermore, the TG accumulation markedly increased by siRNA-mediated knockdown of CAV1 in AML12 cells. By bioinformatics prediction, AML12 cells were transfected of siRNA-118,306 obviously upregulated miR-295-5p. Transfection of miR295-5p mimics significantly increased TG accumulation and obviously suppressed the target CAV1.
\end{abstract}

Conclusions: The results revealed that IncRNA Gm12664-001 attenuated hepatic lipid accumulation through negatively regulating miR-295-5p and enhancing CAV1 expression in AML12 cells.

Keywords: Nonalcoholic fatty liver, Gm12664-001, miR-295-5p, CAV1

\section{Introduction}

Non-alcoholic fatty liver (NAFLD) is a kind of clinical pathological syndrome characterized by excess deposition of fat in liver, which is caused by an imbalance between hepatic synthesis and breakdown of fats, as well as free fatty acids (FFAs) storage and disposal [1]. High fat dietary is closely associated with NAFLD and NAFLD related diseases, including dyslipidemia, body fat deposition, type 2 diabetes mellitus, and thus often regarded as atypical hepatic manifestation of the metabolic syndrome [2]. Furthermore, NAFLD can progress from hepatic steatosis to steatohepatitis, resulting in an increased susceptibility of cirrhosis and hepatocellular carcinoma

\footnotetext{
* Correspondence: liying_helen@163.com

${ }^{1}$ Department of Nutrition and Food Hygiene, Public Health College, Harbin Medical University, Harbin 150086, China

Full list of author information is available at the end of the article
}

[3]. Therefore, correcting and decreasing the factors contributing to excessive accumulation of body fat, have been considered effective strategies to improve NAFLD.

Recently, long non-coding RNAs (lncRNAs) are RNA transcripts consisting of more than $200 \mathrm{bp}$ in length and lack protein-coding capacity [4]. Acting as important regulatory molecules, lncRNAs show moderately evolutional conservation and specific transcription and have roles in transcriptional regulation, epigenetic gene regulation, and disease development, such as NAFLD [5]. The report showed that a novel function of IncRNA SRA in promoting hepatic steatosis through repression of ATGL expression [6]. A conserved IncRNA MRAK052686, was found strongly correlated with the antioxidant factor Nrf2, and both genes were downregulated by the steatotic liver [7].

MicroRNAs (miRNAs) are $\sim 22 \mathrm{nt}$ non-coding RNA molecules that play important roles in post-transcriptional 
regulation in plants and animals [8]. Recent research has demonstrated that miRNA are able to regulate adipocyte differentiation, lipid metabolism, and glucose stimulated insulin secretion to exert influence on metabolic pathways that might be also involved in the pathogenesis of NAFLD [9-12]. They have important roles in mammalian development and human diseases such as NAFLD. The study indicated miR-27a is involving in lipid metabolism and inhibits lipid droplets formation in rat hepatic stellate cells [13]. The findings identify miR-132 as a key regulator of hepatic lipid homeostasis [14], miR-29 involved in lipogenic programs of liver [15], and miR-30 regulating hepatic lipoprotein secretion [16]. Also reported as suppressed are miR-29c in diet-induced NASH and miR-21 [17], miR-29c and miR-451 in livers of $o b / o b$ mice with fatty liver [18]. In addition, lncRNAs were described to compete for miRNA binding, thereby modulating the derepression of miRNA targets [19].

Therefore, we hypothesized that there might also be certain lncRNA regulating steatosis formation in NAFLD through negatively regulating miRNAs expression and directly targeting downstream molecules. In this study, we performed systematical analyses on hepatic expression profiles of lncRNAs and miRNAs in a high-fat diet (HFD)-induced steatotic animal model. We found out miR-295-5p expression was associated with negatively modulated by lncRNA Gm12664-001, the target gene of Gm12664-001, CAV1, was also the target of miR-295$5 p$. Then, we further investigated the mechanisms of lncRNA Gm12664-001 could regulate lipid droplets and triglyceride (TG) level in mice hepatocytes by negatively modulating miR-295-5p and directly recognizing and depressing the expression of CAV1, which contributes to the pathogenesis of steatosis formation in NAFLD and might provide a potential novel therapeutic target for the treatment of NAFLD.

\section{Methods \\ Animals}

Eight-week-old male C57BL/6 mice (40 mice) were provided by the Vital River Laboratories (Beijing, China) for the experiments. All mice were acclimated for 1 week before initiation of the experiment and maintained on a 12/ $12 \mathrm{~h} \mathrm{light/dark}$ cycle with free access to food and water. The animals were divided to the following two groups (20 mice per group) including normal control (NC) group and high fat diet (HFD) group for 8 weeks, the composition of diets in Additional file 1: Table S1. The liver was collected for microarray analysis after 8 weeks of feeding. Experimenters were blind to group assignment and outcome assessment. Animal studies complied with the guidelines of the Harbin Medical University's Regulations of Animal Experiments and were approved by the Animal Experiment Committee of the Harbin Medical University.

\section{Microarrays of miRNAs and IncRNAs}

The total RNA was extracted from total 6 mice $(3$ mice per group at 3 replicates) using the TRIzol reagent (Invitrogen, Carlsbad, CA) according to the manufacturer's protocol. The IncRNAs microarray analysis was performed by KangChen Bio-tech (Shanghai, China). Briefly, the RNA was labeled and hybridized to the Mouse LncRNA Array v2.0 (Arraystar, Rockville, USA), according to Quick Amp Labeling Kit and Gene Expression Hybridization Kit (Agilent Technology, Santa Clara, USA). After washing, the arrays were scanned by the Agilent Microarray Scanner. Agilent Feature Extraction software (version 11.0.1.1) was used to analyze the array images. Quantile normalization and raw data processing were performed using the GeneSpring GX v11.5.1 software package (Agilent Technologies).

\section{Cell culture, treatment and transfection}

Alpha mouse liver 12 (AML12) cells (ATCC, Manassas, VA, USA) were cultured as monolayers in Dulbecco's modified Eagle's medium/F12 (GIBCO BRL) supplemented with 10\% (v/v) fetal bovine serum (PAA Laboratories, Pasching, Austria). The medium contained HEPES $(15 \mathrm{mmol} / \mathrm{L})$, Lglutamine $(2.4 \mathrm{mmol} / \mathrm{L})$, pyridoxine hydrochloride $(2.4$ $\mathrm{mmol} / \mathrm{L})$, dexamethasone $(40 \mathrm{ng} / \mathrm{mL}), \mathrm{NaHCO} 3(1.2 \mathrm{~g} / \mathrm{L})$, penicillin $(100 \mathrm{IU} / \mathrm{mL})$ and streptomycin $(100 \mu \mathrm{g} / \mathrm{mL})$, supplemented with ITS (containing $0.005 \mathrm{mg} / \mathrm{mL}$ insulin, $0.005 \mathrm{mg} / \mathrm{mL}$ transferrin and $5 \mathrm{ng} / \mathrm{mL}$ selenium). Cells were grown under an atmosphere of $5 \%(\mathrm{v} / \mathrm{v})$ $\mathrm{CO}_{2}$ in air at $37^{\circ} \mathrm{C}[20]$.

Stearic acid and Palmitic acid (Sigma, St Louis, MO, USA) were prepared as previously described [21]. Briefly, stearic acid (SA) or palmitic acid (PA) complex with BSA (3 mM fatty acid: $1.5 \mathrm{mM}$ BSA) was dissolved in ethanol and saponified with sodium hydroxide. After the sodium salt was dried, the sodium salt was re-suspended in saline heated $80^{\circ} \mathrm{C}$ until it completely dissolved. When the solution was warm, 20\% (w/v) BSA was added and the mixture was stirred at $50{ }^{\circ} \mathrm{C}$ for $4 \mathrm{~h}$. Then, the complex was sterilized by filtering for further usage.

AML12 cells were treated with 0, 50, 100, 200, 400 and $800 \mu \mathrm{M}$ SA or PA for $24 \mathrm{~h}$. Meanwhile, AML12 cells were also exposed to $300 \mu \mathrm{M}$ SA or $500 \mu \mathrm{M}$ PA for $24 \mathrm{~h}$. The related indicators were measured after fatty acids treatment.

Small-interfering RNAs (siRNA) against Gm12664-001 was purchased from Santa Cruz Biotechnology (Santa Cruz, CA), and AML12 cells were transfected with siRNA118306. The scrambled sequence was used as a negative control (NC). MiR-295-5p mimic and miR-183-5p inhibitor were synthesized by RiboBio Co. (Guangzhou, China). The AML12 cells were transfected with $50 \mathrm{nM}$ of miR-295-5p mimic or $100 \mathrm{nM}$ miR-183-5p inhibitor using Lipofectamine2000 (Invitrogen) according to the manufacturer's instructions. 


\section{Cell viability}

After given $0-800 \mu \mathrm{mol} / \mathrm{L}$ SA or PA for $24 \mathrm{~h}$, removing the culture, $200 \mu \mathrm{L}$ of phosphate buffered saline (PBS) buffer for flushing. Then, $20 \mu \mathrm{L}$ of MTT solution $(5 \mathrm{mg} / \mathrm{mL}$ in PBS as the stock solution) was added to the wells, and the plate was incubated for $4 \mathrm{~h}$ at $37^{\circ} \mathrm{C}$ in the dark. The culture was removed, and $150 \mu \mathrm{L}$ of DMSO was added to each well and left for $10 \mathrm{~min}$ at room temperature. The percentage of viable cells was measured using a plate reader at $570 \mathrm{~nm}$ [22].

\section{Real time-PCR}

Total RNAs with miRNAs was isolated with the miRNeasy Mini Kit (Qiagen, Hilden, Germany) according to the manufacturer's protocol. The miScript SYBR Green PCR Kit (Qiagen) was used to perform qPCR, and the expression level of U6 was used as an internal control. Additionally, all primers were purchased from Qiagen.

\section{Target prediction and luciferase activity assay}

The pmiR-RB-REPORT ${ }^{\mathrm{m}}$ dual luciferase reporter vector carrying the 3'-UTR of miR-295-5p target gene CAV1 was constructed (Ribio Co., Guangzhou, China). AML12 cells were co-transfected with $50 \mathrm{ng}$ of recombinant plasmid, $100 \mathrm{nM}$ of miR-295-5p mimic and mimic NC. After $24 \mathrm{~h}$ of transfection, the luciferase activities were measured with a dual luciferase reporter assay kit on a luminometer (GloMaxTM 20/20, Promega).

\section{Hematoxylin eosin (HE) staining}

HE staining was conducted according to routine procedure. In brief, after deparaffinization and rehydration, $5 \mu \mathrm{m}$ longitudinal sections were stained with hematoxylin solution for $5 \mathrm{~min}$ followed by 5 dips in 1\% acid ethanol $(1 \% \mathrm{HCl}$ in $70 \%$ ethanol) and then rinsed in distilled water. Then the sections were stained with eosin solution for $3 \mathrm{~min}$ and followed by dehydration with graded alcohol and clearing in xylene [23].

\section{TG determination}

AML12 cells were transfected with siRNA-118,306, mimicmiR-295-5p, inhibitor-miR-183-5p or siRNA-CAV1 for 48 $h$. The cells were then washed three times with ice-cold PBS and stained with BODIPY ${ }^{\circ}$ (Molecular Probes, Eugene, OR, USA) staining solution (diluted with PBS, 1:1000) for $30 \mathrm{~min}$ at room temperature. Then cells were then washed again with PBS three times, to remove unbound solution [24]. TG was assayed using commercial kits using enzymatic methods (APPLYGEN, Beijing, China), according to the manufacturers' instructions.

\section{Western blot}

Western blot was performed as previously described [25] using the following primary antibodies: ATGL, HSL, FAS and CAV1 (Cell Signaling Technology, Danvers, MA, USA) as well as DGAT2 and $\beta$-actin (Santa Cruz Biotechnology, Dallas, TX, USA). Anti-rabbit alkaline phosphatase-conjugated antibody (Promega, Madison, WI, USA) was used as a secondary antibody.

\section{Statistical analysis}

Results were presented as mean \pm standard deviation. The SPSS 18.0 software was used for all statistical analyses. Statistical analyses were conducted by $t$ test or one-way ANOVA, $P<0.05$ was considered to indicate a significant difference.
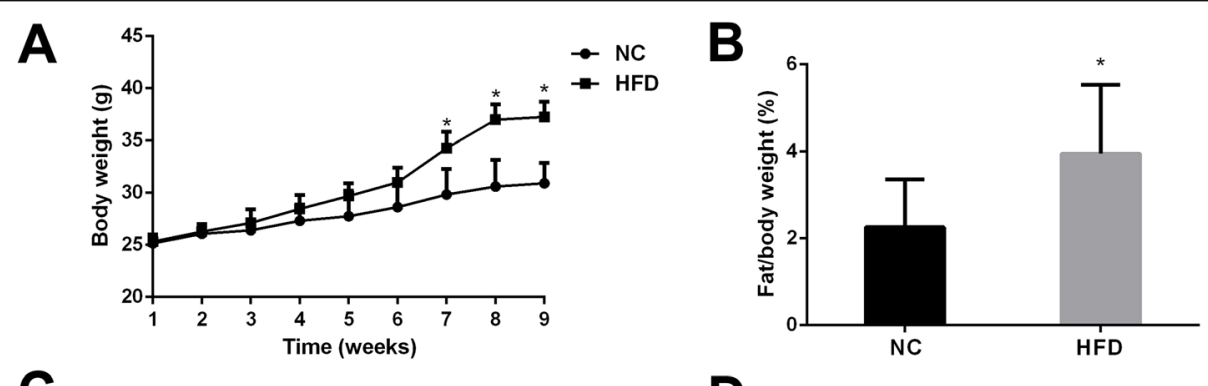

D
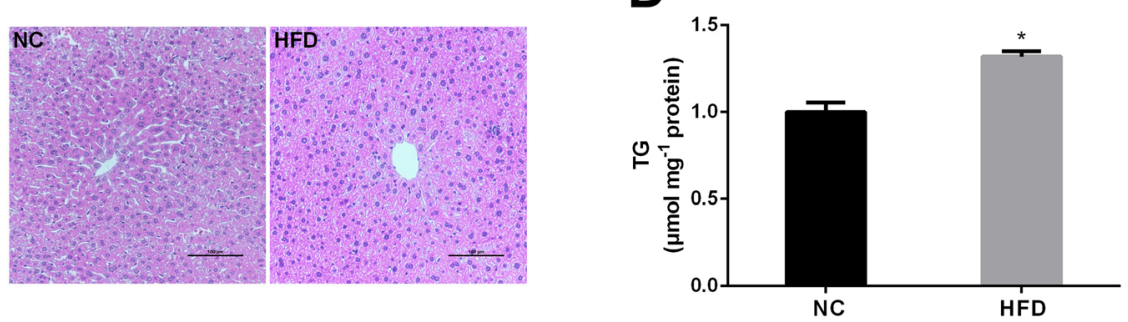

Fig. 1 Effects of HFD treatment on lipid accumulation in mice. The body weight (a), body fat rate (b), HE staining (c) and TG level (d) after the treatment with HFD in mice. The experiments were repeated 3 times. Data are presented as means $\pm \mathrm{SD}(n=3)$. ${ }^{*} P<0.05$, compared with normal control 


\section{Results}

High fat diet contributes to lipid accumulation in mice Compared with normal control, the mice in high fat diet group increased body weight and body fat rate in mice (Fig. 1a, b). The morphological changes of liver were observed by using HE staining (Fig. 1c). HFD treatment markedly observed hepatic fatty degeneration with primarily fat vacuoles was visible, and significantly increased TG level (Fig. 1d), indicating that the high fat diet induced successfully fat mice model with TG accumulation in liver $(P<0.05)$.

\section{LncRNA Gm12664-001 determination}

Our microarray data identified 364 upregulated and 387 downregulated IncRNAs, and 29 upregulated and 13 downregulated miRNAs were differentially expressed found in NAFLD samples as compared with the normal liver samples. Among them, IncRNA Gm12664-001 (ENSMUST00000118306) was found to be the most significantly upregulated, and were further verified by RT-PCR (Fig. 2a).

In this study, AML12 cells were treated with 0$800 \mu \mathrm{M}$ SA or PA for $24 \mathrm{~h}$ to evaluate cell viability by MTT assay (Fig. 2b, c). We found that the level of IncRNA Gm12664-001 was markedly increased after treatment with $300 \mu \mathrm{M}$ SA or $500 \mu \mathrm{M}$ PA for $24 \mathrm{~h}$ in AML12 cells (Fig. 2d). LncRNA Gm12664-001 level was markedly decreased after transfection with Gm12664001 siRNA (siRNA-118,306) for $48 \mathrm{~h}$ in AML12 cells by Real-time PCR (Fig. 2e).

Effects of IncRNA Gm12664-001 on TG level and the key protein expressions involved in lipid metabolism in AML12 cells

Lipid droplets and TG level were significantly enhanced after transfection of siRNA-118,306 in AML12 cells (Fig. 3a, b). Moreover, the results suggest that the transfection with siRNA-118,306 has no effect on protein
A

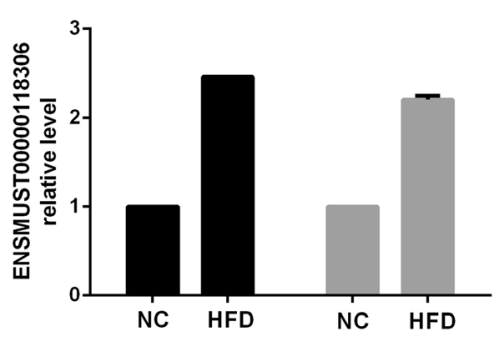

C

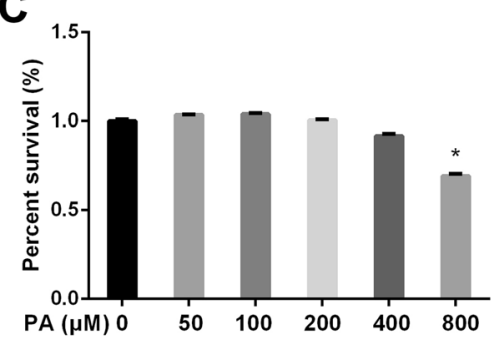

E

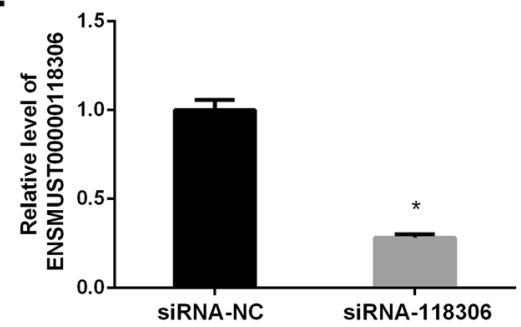

B

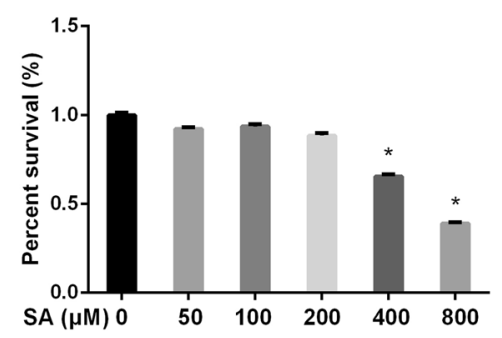

D

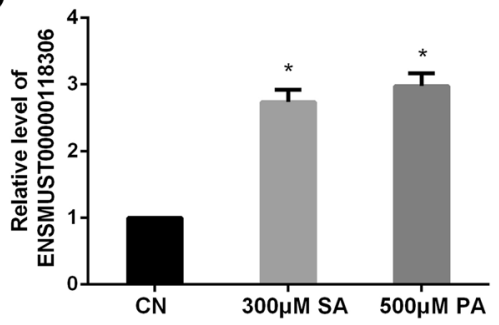

F. 2 The validation of IncRNAs. The levels of IncRNA Gm12664-001 (ENSMUST00000118306) by microarray and RT-PCR in liver (a). The cytotoxicity of 0, 50, 100, 200, 400, $800 \mu \mathrm{M} \mathrm{SA} \mathrm{(b)} \mathrm{or} \mathrm{PA} \mathrm{(c)} \mathrm{for} 24 \mathrm{~h}$ with MTT assay in AML12 cells. The levels of Gm126604-001 after treatment with $300 \mu \mathrm{M}$ SA or $500 \mu \mathrm{M}$ PA in AML12 cells (d). The level of Gm126604-001 (e) after the transfection with siRNA-118,306 in AML12 cells. The experiments were repeated 3 times. Data are presented as means \pm SD $(n=3)$. $P<0.05$, compared with control or siRNA-NC 
A

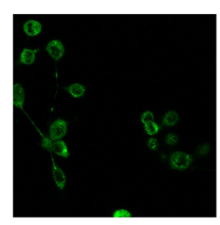

SiRNA-NC

C
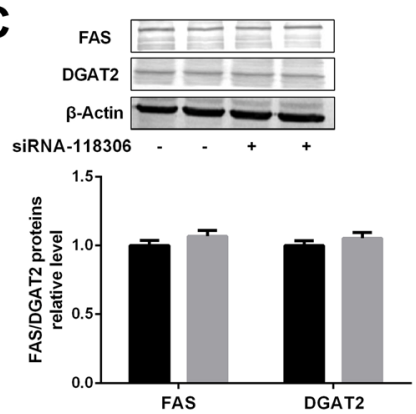

E

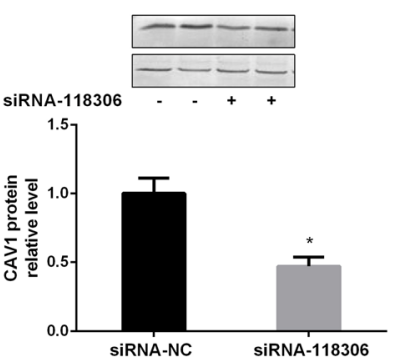

B

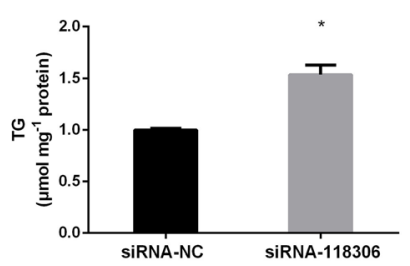

D
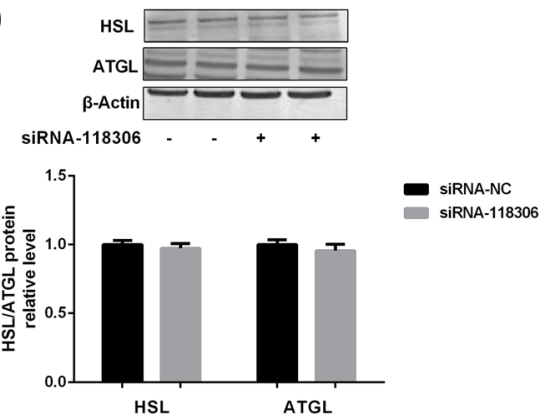

F

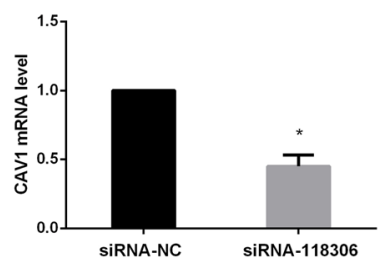

Fig. 3 The TG level and protein expressions involved in lipid metabolism in AML12 cells. The lipid droplets (a) and TG level (b) after transfection of siRNA-118,306 in AML12 cells. The expressions of FAS and DGAT2 (c), HSL and ATGL (d) after the transfection of siRNA-118,306 for $48 \mathrm{~h}$ in AML12 cells. The CAV1 protein expression (e) and mRNA level (f) after transfection of siRNA-118,306 for $48 \mathrm{~h}$ in AML12 cells. The experiments were repeated 3 times. Data are presented as means $\pm \operatorname{SD}(n=3) .{ }^{*} P<0.05$, compared with siRNA-NC

expressions involved the synthesis and decomposition of TG, such as FAS, DGAT2, HSL and ATGL in AML12 cells (Fig. 3c, d). However, CAV1 protein and mRNA levels were mainly involved in transmembrane transport and lipid accumulation markedly suppressed after the transfection of siRNA-118,306 for $48 \mathrm{~h}$ in AML12 cells (Fig. 3e, f).

\section{Effects of CAV1 protein on TG accumulation in AML12 cells}

The results suggest that the level of CAV1 mRNA significantly decreased after the transfection of siRNA-CAV1 in AML12 cells (Fig. 4a). The lipid droplets and TG level were obviously increased after transfection of siRNACAV1 in AML12 cells (Fig. 4b, c, $P<0.05$ ).

\section{MiR-295-5p contributes to lipid accumulation and CAV1 expression in AML12 cells}

By bioinformatics prediction, we found out 6 miRNAs associated with lncRNA Gm12664-001 among 42 differentially expressed miRNAs. As we expected, AML12 cells were transfected with siRNA-118,306 significantly decreased Gm12664-001 and miR-183-5p levels, and obviously enhanced miR-295-5p expression (Fig. 5a).

Transfection of miR-295-5p mimics significantly enhanced lipid droplets and TG level (Fig. 5b, c), but miR183-5p inhibitor did not change in AML12 cells (Fig. 5d, e). Moreover, overexpression of miR-295-5p obviously repressed CAV1 protein expression in AML12 cells, and luciferase assay evidently inhibited luciferase activity in AML12 cells further verified that CAV1 was the target of miR-295-5p (Fig. 5f, g).

\section{Discussion}

NAFLD is a kind of chronic liver disease, whose spectrum ranges from simple fatty liver, with benign prognosis, to a potentially progressive form, NASH, which may lead to liver fibrosis and cirrhosis, resulting in increased morbidity and mortality [26]. Without effective medical interventions for complete reverse of NAFLD, it needs to urgently investigate the potential molecular mechanisms of NAFLD to provide a novel the therapeutic strategy for people suffering from NAFLD. 


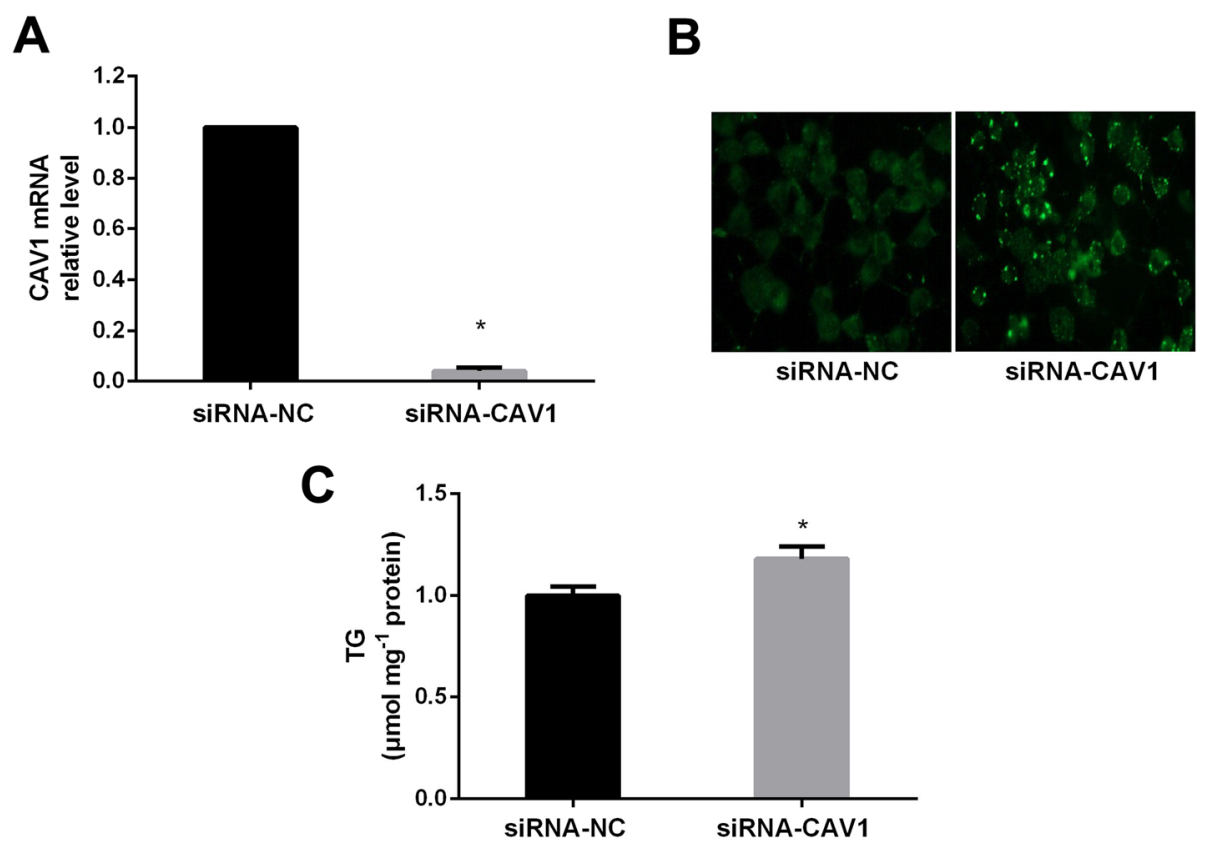

Fig. 4 Effects of CAV1 expression on TG accumulation in AML12 cells. The CAV1 mRNA level (a), lipid droplets (b) and TG level (c) after transfection of siRNA-CAV1 in AML12 cells. The experiments were repeated 3 times. Data are presented as means \pm SD $(n=3) .{ }^{*} P<0.05$, compared with siRNA-NC

In our studies, we found out HFD induced mice markedly increased body weight, and body fat rate compared with normal control. Additionally, the liver is a major determinant of whole body maintenance of lipid homeostasis and it's responsible for the synthesis and secretion of fatty acids and TG. Our results suggest that HFD treatment markedly observed hepatic fatty degeneration with primarily fat vacuoles was visible and significantly increased TG level, indicating that the HFD induced successfully high fat mice model with TG accumulation in liver.

LncRNAs are shown to be evolutionally conserved and have roles in transcriptional regulation, epigenetic gene regulation, and disease development, such as embryogenesis, cellular differentiation, tumorigenesis, and hepatic lipid metabolism [27]. LncRNAs were considered to compete for miRNA binding, thereby modulating the expression of miRNA targets. By analyzing gene expression profiles, we identified 751 lncRNAs and 42 miRNAs, which displayed differential expression patterns among the normal control and high fat diet treated mice. We delineated several modules and identified numerous significant genes exhibiting a wide association with liver metabolism and NAFLD-related functions. A significant example of these lncRNAs is Gm12664-001. Our results suggest that lncRNA Gm12664-001 level was markedly up-regulated after $200 \mu \mathrm{M}$ SA or $300 \mu \mathrm{M}$ PA-induced TG accumulations in AML12 cells. This result indicated that Gm12664-001 might play a vital role in NAFLD pathological processes. Thus, the outcome might help us provide a new sally port to investigate the underlying molecular mechanisms of NAFLD.

Caveolin-1 (CAV1) is a structural protein of caveolae mainly involved in lipid homeostasis and endocytosis [28]. The reports demonstrate that CAV1 plays an important role in the modulation of lipid metabolism during liver regeneration, and lack of CAV1 alters hepatocyte energy metabolism homeostasis under physiological and pathological conditions [29]. Our results indicated that lipid droplets and TG level were significantly increased after transfection with siRNA-CAV1 in AML12 cells. Additionally, the studies have shown that CAV1 knockout mice display several metabolic abnormalities, including dyslipidemia, hyperglycemia and IR in liver and/or fat tissues $[30,31]$. These data further suggest that CAV1 gene was the key molecule of lipid metabolism pathways.

By bioinformatics prediction, we found a strong coexpression of Gm12664-001 and miR-295-5p was demonstrated by either microarray analysis or the experimental data from HFD-fed animals. This implies a potential role of miR-295-5p was negatively modulated by IncRNA Gm12664-001, and significantly decreased lipid accumulation in liver, which associated with CAV1, the gene that are critically involved in NAFLD. Moreover, overexpression of miR-295-5p markedly repressed CAV1 protein expression, and luciferase assay obviously inhibited luciferase activity in AML12 cells further verified that CAV1 was also the target of miR-295-5p. 


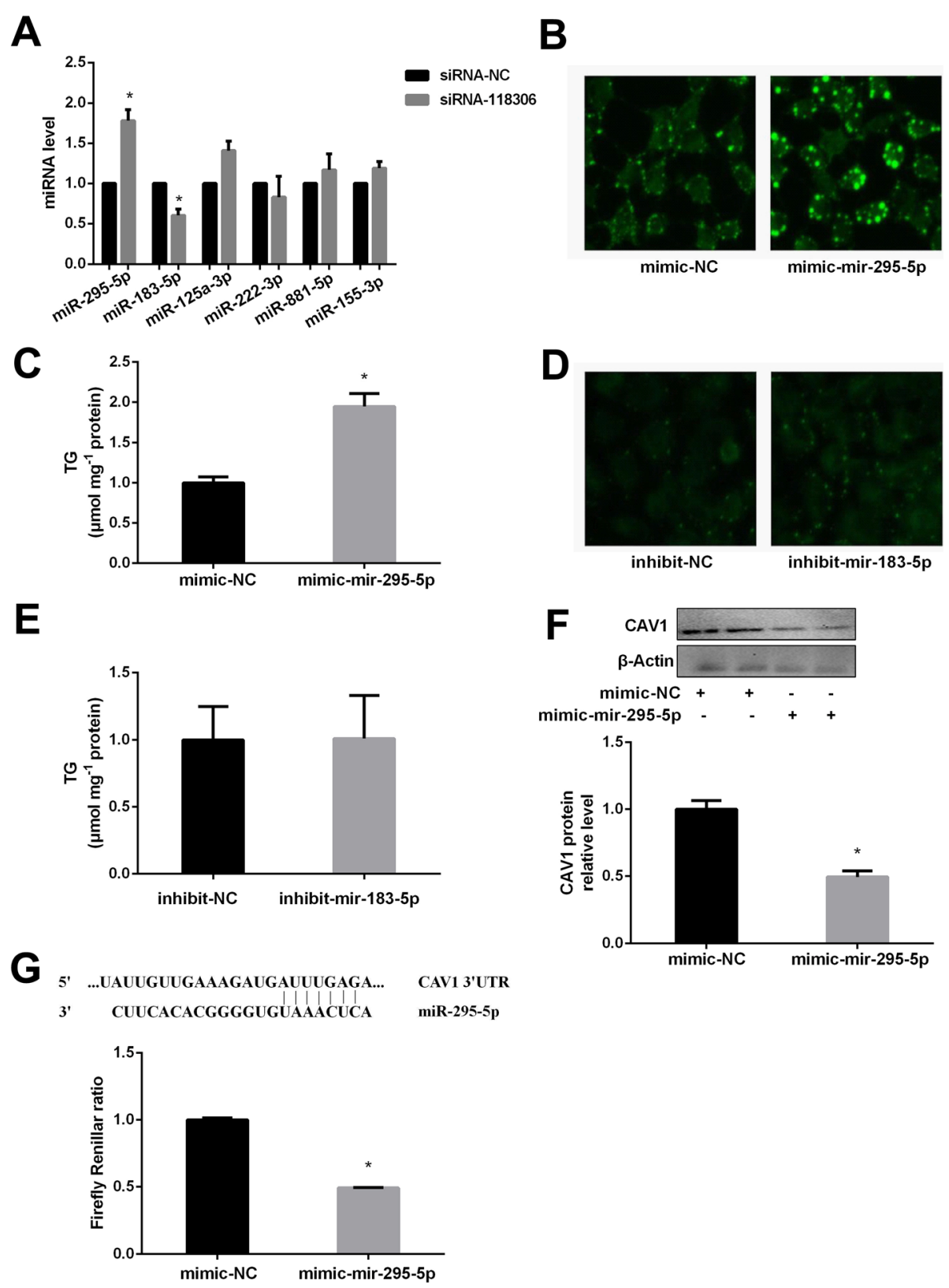

Fig. 5 Effects of miR-295-5p on TG accumulation and CAV1 expression in AML12 cells. The expressions of miRNAs (a) after the transfection with siRNA-118,306 in AML12 cells. The lipid droplets and TG level after transfection of mimic-miR-295-5p (b, c) or inhibit-miR-183-5p (d, e) in AML12 cells. The expression of CAV1 (f) and the effects of miR-295-5p on 3'-UTR of CAV1 by luciferase activity assay ( $\mathbf{g}$ ) after transfection of miR-295-5p mimic in AML12 cells. The experiments were repeated 3 times. Data are presented as means \pm SD $(n=3)$. $P<0.05$, compared with mimic-NC

\section{Conclusion}

The findings of our study clearly showed that lncRNA Gm12664-001 negatively modulating miR-295-5p and repressing the target of CAV1 expression and lowering TG level in liver suggest that lncRNA Gm12664-001 may play important roles in NAFLD by affecting lipid accumulation, which provides possible mechanisms and warrants further investigations.

\section{Supplementary information}

Supplementary information accompanies this paper at https://doi.org/10. 1186/s12986-020-0430-z.

Additional file 1: Table S1. The composition of high-fat diet and basic diet.

\section{Abbreviations}

AML12: Alpha mouse liver 12; CAV1: Caveolin-1; FFAs: Free fatty acids; HE: Hematoxylin eosin; HFD: High fat diet; LncRNAs: Long non-coding RNAs; 
MiRNAs: MicroRNAs; NAFLD: Non-alcoholic fatty liver; RER: Respiratory exchange rate; TG: Triglycerides

\section{Acknowledgements \\ Not applicable}

\section{Authors' contributions}

YL designed the study; QZ wrote the paper; QZ, JW, HL and YZ accomplished experimental studies. XC and JY put forward suggestions for the expansion and modification of manuscript. All authors read and approved the final manuscript.

\section{Funding}

This study was supported by the Applied Technology Research and Development Plan of Heilongjiang Province (GA18C005) and the National Natural Science Foundation of China (81673153).

\section{Availability of data and materials}

All data generated or analysed during this study are included in this published article [and its supplementary information files].

\section{Ethics approval and consent to participate}

Not applicable

\section{Consent for publication}

Not applicable

\section{Competing interests}

The authors declare that they have no competing interests.

\section{Author details}

${ }^{1}$ Department of Nutrition and Food Hygiene, Public Health College, Harbin Medical University, Harbin 150086, China. ${ }^{2}$ Department of Public Health College, Kunming Medical University, Kunming 650550, China. ${ }^{3}$ School of Public Health, Ningxia Medical University, Yinchuan 750004, China.

Received: 8 April 2019 Accepted: 20 January 2020

Published online: 04 February 2020

\section{References}

1. Mazzoccoli G, Vinciguerra M, Oben J, Tarquini R, De Cosmo S. Non-alcoholic fatty liver disease: the role of nuclear receptors and circadian rhythmicity. Liver Int. 2014:34:1133-52

2. Mokdad AH, Ford ES, Bowman BA, Dietz WH, Vinicor F, Bales VS, Marks JS. Prevalence of obesity, diabetes, and obesity-related health risk factors, 2001. JAMA. 2003;289:76-9.

3. Kahali B, Halligan B, Speliotes EK. Insights from genome-wide association analyses of nonalcoholic fatty liver disease. Semin Liver Dis. 2015;35:375-91.

4. Wilusz JE, Sunwoo H, Spector DL. Long noncoding RNAs: functional surprises from the RNA world. Genes Dev. 2009;23:1494-504.

5. Zhang M, Sun W, Zhou M, Tang Y. MicroRNA-27a regulates hepatic lipid metabolism and alleviates NAFLD via repressing FAS and SCD1. Sci Rep. 2017;7:14493.

6. Chen G, Yu D, Nian X, Liu J, Koenig RJ, Xu B, Sheng L. LncRNA SRA promotes hepatic steatosis through repressing the expression of adipose triglyceride lipase (ATGL). Sci Rep. 2016;6:35531.

7. Yuan X, Wang J, Tang X, Li Y, Xia P, Gao X. Berberine ameliorates nonalcoholic fatty liver disease by a global modulation of hepatic mRNA and IncRNA expression profiles. J Transl Med. 2015;13:24.

8. Guo L, Lu Z. Global expression analysis of miRNA gene cluster and family based on isomiRs from deep sequencing data. Comput Biol Chem. 2010;34:165-71.

9. Hartig SM, Hamilton MP, Bader DA, McGuire SE. The miRNA Interactome in metabolic homeostasis. Trends Endocrinol Metab. 2015;26:733-45.

10. Rottiers $V$, Naar AM. MicroRNAs in metabolism and metabolic disorders. Nat Rev Mol Cell Biol. 2012;13:239-50.

11. Poy MN, Spranger M. Stoffel M: microRNAs and the regulation of glucose and lipid metabolism. Diabetes Obes Metab. 2007;9(Suppl 2):67-73.

12. Zarfeshani A, Ngo S, Sheppard AM. MicroRNA expression relating to dietaryinduced liver Steatosis and NASH. J Clin Med. 2015;4:1938-50.
13. Ji J, Zhang J, Huang G, Qian J, Wang X, Mei S. Over-expressed microRNA27a and 27b influence fat accumulation and cell proliferation during rat hepatic stellate cell activation. FEBS Lett. 2009;583:759-66.

14. Hanin G, Yayon N, Tzur Y, Haviv R, Bennett ER, Udi S, et al. miRNA-132 induces hepatic steatosis and hyperlipidaemia by synergistic multitarget suppression. Gut. 2018;67(6):1124-34.

15. Kurtz CL, Fannin EE, Toth $C L$, Pearson DS, Vickers KC, Sethupathy $P$. Inhibition of miR-29 has a significant lipid-lowering benefit through suppression of lipogenic programs in liver. Sci Rep. 2015;5:12911.

16. Soh J, lqbal J, Queiroz J, Fernandez-Hernando C, Hussain MM. MicroRNA-30C reduces hyperlipidemia and atherosclerosis in mice by decreasing lipid synthesis and lipoprotein secretion. Nat Med. 2013;19:892-900.

17. Pogribny IP, Starlard-Davenport A, Tryndyak VP, Han T, Ross SA, Rusyn I, Beland FA. Difference in expression of hepatic microRNAs miR-29c, miR-34a, miR-155, and miR-200b is associated with strain-specific susceptibility to dietary nonalcoholic steatohepatitis in mice. Lab Investig. 2010;90:1437-46.

18. Li S, Chen X, Zhang H, Liang X, Xiang Y, Yu C, Zen K, Li Y, Zhang CY. Differential expression of microRNAs in mouse liver under aberrant energy metabolic status. J Lipid Res. 2009:50:1756-65.

19. Cesana M, Cacchiarelli D, Legnini I, Santini T, Sthandier O, Chinappi M, Tramontano A, Bozzoni I. A long noncoding RNA controls muscle differentiation by functioning as a competing endogenous RNA. Cell. 2011;147:358-69.

20. Wada W, Medina JJ, Kuwano H, Kojima I. Comparison of the function of the beta(C) and beta(E) subunits of activin in AML12 hepatocytes. Endocr J. 2005;52:169-75.

21. Deng H, Chu X, Song Z, Deng X, Xu H, Ye Y, Li S, Zhang Q, Sun C, Li Y. MicroRNA-1185 induces endothelial cell apoptosis by targeting UVRAG and KRIT1. Cell Physiol Biochem. 2017:41:2171-82.

22. Zhang Q, Yuan H, Zhang C, Guan Y, Wu Y, Ling F, Niu Y, Li Y. Epigallocatechin gallate improves insulin resistance in HepG2 cells through alleviating inflammation and lipotoxicity. Diabetes Res Clin Pract. 2018;142:363-73.

23. Liu H, Zhu R, Liu C, Ma R, Wang L, Chen B, Li L, Niu J, Zhao D, Mo F, et al. Evaluation of decalcification techniques for rat femurs using $\mathrm{HE}$ and Immunohistochemical staining. Biomed Res Int. 2017;2017:9050754.

24. Tavian D, Colombo R. Improved cytochemical method for detecting Jordans' bodies in neutral lipid storage diseases. J Clin Pathol. 2007;60:956-8.

25. Lu N, Li Y, Qin H, Zhang YL, Sun CH. Gossypin up-regulates LDL receptor through activation of ERK pathway: a signaling mechanism for the hypocholesterolemic effect. J Agric Food Chem. 2008;56:11526-32.

26. Marrero JA, Fontana RJ, Su GL, Conjeevaram HS, Emick DM, Lok AS. NAFLD may be a common underlying liver disease in patients with hepatocellular carcinoma in the United States. Hepatology. 2002;36:1349-54.

27. Li P, Ruan X, Yang L, Kiesewetter K, Zhao Y, Luo H, Chen Y, Gucek M, Zhu J, $\mathrm{Cao} \mathrm{H}$. A liver-enriched long non-coding RNA, IncLSTR, regulates systemic lipid metabolism in mice. Cell Metab. 2015;21:455-67.

28. Valasek MA, Weng J, Shaul PW, Anderson RG, Repa JJ. Caveolin-1 is not required for murine intestinal cholesterol transport. J Biol Chem. 2005;280:28103-9.

29. Fernandez-Rojo MA, Restall C, Ferguson C, Martel N, Martin S, Bosch M, Kassan A, Leong GM, Martin SD, McGee SL, et al. Caveolin-1 orchestrates the balance between glucose and lipid-dependent energy metabolism: implications for liver regeneration. Hepatology. 2012;55:1574-84.

30. Razani B, Combs TP, Wang XB, Frank PG, Park DS, Russell RG, Li M, Tang B, Jelicks LA, Scherer PE, Lisanti MP. Caveolin-1-deficient mice are lean, resistant to diet-induced obesity, and show hypertriglyceridemia with adipocyte abnormalities. J Biol Chem. 2002:277:8635-47.

31. Baudrand R, Gupta N, Garza AE, Vaidya A, Leopold JA, Hopkins PN, Jeunemaitre X, Ferri C, Romero JR, Williams J, et al. Caveolin 1 modulates aldosterone-mediated pathways of glucose and lipid homeostasis. J Am Heart Assoc. 2016:5(10).

\section{Publisher's Note}

Springer Nature remains neutral with regard to jurisdictional claims in published maps and institutional affiliations. 